\title{
Toll-like receptor 4 imparts ligand-specific recognition of bacterial lipopolysaccharide
}

\author{
Egil Lien, $, 1,2$ Terry K. Means, ${ }^{3}$ Holger Heine, ${ }^{1}$ Atsutoshi Yoshimura, ${ }^{1}$ Shoichi Kusumoto, ${ }^{4}$ \\ Koichi Fukase, ${ }^{4}$ Matthew J. Fenton, ${ }^{3}$ Masato Oikawa, ${ }^{4}$ Nilofer Qureshi, ${ }^{5}$ Brian Monks, ${ }^{1}$ \\ Robert W. Finberg, ${ }^{6}$ Robin R. Ingalls, ${ }^{1}$ and Douglas T. Golenbock ${ }^{1}$
}

\author{
${ }^{1}$ The Maxwell Finland Laboratory for Infectious Diseases, Boston Medical Center, Boston, Massachusetts 02118, USA \\ ${ }^{2}$ Institute of Cancer Research and Molecular Biology, Norwegian University of Science and Technology, \\ 7489 Trondheim, Norway \\ ${ }^{3}$ Pulmonary Center, Boston University School of Medicine, Boston, Massachusetts 02118, USA \\ ${ }^{4}$ Department of Chemistry, Graduate School of Science, Osaka University, Toyonaka 560-0043, Japan \\ ${ }^{5}$ William S. Middleton Memorial Veterans Hospital, Madison, Wisconsin 53705, USA \\ ${ }^{6}$ Laboratory of Infectious Diseases, Dana-Farber Cancer Institute, Boston, Massachusetts 02115, USA
}

Address correspondence to: Douglas T. Golenbock, The Maxwell Finland Laboratory for Infectious Diseases, 774 Albany Street, Boston, Massachusetts 02118, USA. Phone: (617) 414-7965; Fax: (617) 414-5843; E-mail: Douglas.Golenbock@bmc.org.

Egil Lien's present address is: Institute of Cancer Research and Molecular Biology, Norwegian University of Science and Technology, University Medical Center, 7489 Trondheim, Norway.

Received for publication September 24, 1999, and accepted in revised form December 23, 1999.

\begin{abstract}
Lipopolysaccharide (LPS) is the main inducer of shock and death in Gram-negative sepsis. Recent evidence suggests that LPS-induced signal transduction begins with CD14-mediated activation of 1 or more Toll-like receptors (TLRs). The lipid A analogues lipid IVa and Rhodobacter sphaeroides lipid A (RSLA) exhibit an uncommon species-specific pharmacology. Both compounds inhibit the effects of LPS in human cells but display LPS-mimetic activity in hamster cells. We transfected human TLR4 or human TLR2 into hamster fibroblasts to determine if either of these LPS signal transducers is responsible for the species-specific pharmacology. RSLA and lipid IVa strongly induced NF- $\mathrm{KB}$ activity and IL-6 release in Chinese hamster ovary fibroblasts expressing CD14 (CHO/CD14), but these compounds antagonized LPS antagonists in CHO/CD14 fibroblasts that overexpressed human TLR4. No such antagonism occurred in cells overexpressing human TLR2. We cloned TLR4 from hamster macrophages and found that human THP-1 cells expressing the hamster TLR4 responded to lipid IVa as an LPS mimetic, as if they were hamster in origin. Hence, cells heterologously overexpressing TLR4 from different species acquired a pharmacological phenotype with respect to recognition of lipid A substructures that corresponded to the species from which the TLR 4 transgene originated. These data suggest that TLR4 is the central lipid A-recognition protein in the LPS receptor complex.
\end{abstract}

J. Clin. Invest. 105:497-504 (2000).

\section{Introduction}

Gram-negative bacterial sepsis is a common cause of shock and death (1). Lipopolysaccharide (LPS), a major constituent of the Gram-negative bacterial outer membrane, can trigger a variety of inflammatory reactions, including the release of proinflammatory cytokines and other soluble factors. If produced in excess, these mediators induce the systemic inflammation that causes end-organ damage, sepsis, and death. The LPS molecule is complex, consisting of a polysaccharide, a core oligosaccharide, and a highly conserved lipid A portion. The lipid A moiety is responsible for the toxic proinflammatory properties of LPS, and is therefore a target for the development of medical therapies for the treatment of sepsis (2).

Multiple mammalian receptors for endotoxin have been identified over the last decade. The most important of these is the glycosylphosphatidylinositol-linked protein CD14 (3). Although there is little doubt that CD14 binds LPS and initiates signal transduction,
CD14 is not by itself capable of initiating a transmembrane activation signal. First, because CD14 lacks a transmembrane domain, it has no intrinsic signaling capabilities. Second, LPS receptor antagonists inhibit the effects of LPS at concentrations that are too low to block LPS binding to CD14 $(4,5)$, suggesting that blockade of CD14 is not the mechanism of receptor inhibition. This has led many to postulate that LPS/CD14 complexes interact with a transmembrane receptor that is responsible for ligand specificity and signal transduction (6-8).

Strong evidence for the existence of a CD14-associated signal transducer comes from the characterization of lipid A-like molecules that antagonize LPS both in vitro and in vivo (9). These include the lipid A analogues lipid IVa and Rhodobacter sphaeroides lipid A (RSLA). RSLA and lipid IVa are both potent LPS antagonists in LPS-responsive human cells (10). Curiously, in native hamster macrophages, both compounds are LPS mimetics (6). The pharmacology of 
these drugs is even more complicated in mice, with RSLA acting as an LPS antagonist, whereas lipid IVa is an LPS mimetic.

The prominent role of CD14 in binding and initiating LPS signals made this receptor an obvious candidate as the molecule responsible for these species-specific effects. Yet, molecular genetic studies in human, hamster, and mouse cell lines that were heterologously transfected with mouse or human CD14 demonstrated that the origin species of CD14 was irrelevant to the observed pharmacology of RSLA and lipid IVa (6). These studies implied that the gene product responsible for the species-specific pharmacology of LPS would be the lipid A-recognition component of the LPS receptor complex.

Recently, members of the Toll receptor family have been implicated in LPS signaling. Toll, a type I transmembrane receptor with homology to the intracellular portion of the IL-1 receptor, was initially identified as a receptor involved in the embryonic development of Drosophila melanogaster, in which it controls dorsoventral polarization. Subsequently, it was demonstrated that Toll and its homologues also control the induction of antimicrobial factors in response to infection (11-13). A family of mammalian Toll-like receptors (TLRs) has also been described (14). Two members of this family, TLR2 and TLR4, have been identified as possible LPS signaling receptors (15-20).

We reasoned that the biology of the true endotoxin receptor should account for all aspects of the complex pharmacology that has been described for LPS. In light of our previous findings concerning the species-specific effects of the LPS antagonists, we hypothesized that if a TLR were the major component of the LPS signaling complex, then it would also have to account for the species-specific pharmacology of RSLA and lipid IVa. To test this hypothesis, we transfected human and hamster constructs for TLRs into a Chinese hamster ovary K1 (CHO-K1) fibroblast line expressing CD14

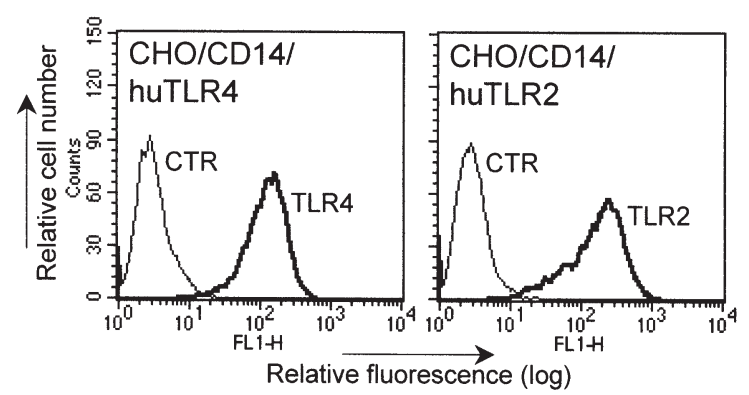

Figure 1

Expression of human TLR4 and TLR2 transgenes in CHO/CD14 cells. $\mathrm{CHO} / \mathrm{CD} 14$ cells that were stably transfected with human TLR4 or human TLR2 were labeled with $10 \mu \mathrm{g} / \mathrm{mL}$ of the mAbs HTA125 (TLR4), TL2.1 (TLR2), or a control antibody (CTR, mouse IgG; Sigma-Aldrich), followed by incubation with anti-mouse IgG FITC (Sigma-Aldrich). The cells were subjected to flow cytometry analysis on as described (42). Relative cell number is shown on the $y$-axis and relative fluorescence is shown on the $x$-axis.
(CHO/CD14), and a human monocytic cell line, THP1. The results demonstrated a dramatic shift in phenotype based on the origin species of TLR 4 expressed. In contrast, expression of TLR2 had no effect on these species-specific activities of the compounds tested. We conclude that TLR4 functions to alert immune cells to the presence of LPS, and is responsible for the speciesspecific recognition of lipid A structures. This ligandspecific recognition strongly supports the concept that LPS directly binds to TLR4. The challenge for the future is to identify this presumed binding site; this accomplishment will be critical for the development of anti-endotoxin therapies for the medical treatment of human septic shock.

\section{Methods}

Reagents. PBS, Ham's F12 medium, RPMI-1640 medium, and trypsin-versene mixture were from BioWhittaker Inc. (Walkersville, Maryland, USA). Low-endotoxin FBS was from Summit Biotechnology (Greeley, Colorado, USA). Ciprofloxacin was a gift from Miles Pharmaceuticals (West Haven, Connecticut, USA). Hygromycin B was purchased from Calbiochem-Novabiochem Corp. (San Diego, California, USA), puromycin was from Sigma-Aldrich (St. Louis, Missouri, USA), and G418 came from GIBCO BRL (Gaithersburg, Maryland, USA). Salmonella minnesota R595 LPS and RSLA were as described previously (10); alternatively, Re595 LPS from Sigma-Aldrich was used. Synthetic lipid A (Escherichia coli-like, also known as compound 506) and the tetraacyl lipid A precursor known as lipid IVa (compound 406) were synthesized as described (21). Human IL-1 $\beta$ and IL- 6 were purchased from Genzyme Pharmaceuticals (Cambridge, Massachusetts, USA). Antibodies for reporter cell assay (CD25) were purchased from Becton Dickinson Immunocytometry Systems (San Jose, California, USA). Specific mAbs against TLR2 (mAb TL2.1) have been reported elsewhere (22). Anti-TLR4 mAb HTA125 (23) was a gift from K. Miyake (Saga Medical School, Saga, Japan). Plasmids encoding the cDNA for human TLR2 (huTLR2) or TLR4 (huTLR4) in a pFLAG-CMV-1 vector were the gifts of M. Rothe, Tularik Inc. (South San Francisco, California, USA) (16). Mouse TLR2 (moTLR2) was cloned from a cDNA library as described previously (24); this cDNA was capable of mediating LPS effects in transfected HEK293 cells (24). An untagged version of huTLR4 (hToll; ref. 25) in the vector pcDNA3.1 was a gift from C. Janeway and R. Medzhitov (Yale University, New Haven, Connecticut, USA).

Cell lines. The $\mathrm{CHO} / \mathrm{CD} 14$ reporter line (clone 3E10; ref. 26) is a stably transfected CD14-positive CHO cell line that expresses inducible membrane CD25 (Tac antigen) under transcriptional control of the human E-selectin promoter. The promoter fragment chosen contains an essential NF- $\mathrm{KB}$ binding site (27). LPS, IL1 , and TNF- $\alpha$ all activate NF- $\kappa B$ in these cells, resulting in a 3 - to 10-fold increase in the surface expression 
of CD25. The $\mathrm{CHO} / \mathrm{CD} 14 /$ huTLR2 reporter cell lines were constructed by stable cotransfection of $\mathrm{CHO} / \mathrm{CD} 14$ with the cDNA for human TLR2 and pcDNA3 (Invitrogen Corp., Carlsbad, California, USA), as described (28). The CHO/CD14/huTLR4 reporter cell lines were derived in the same manner, except that a puromycin resistance plasmid (pRc/RSV; gift of R. Kitchens, University of Texas, Southwestern Medical Center, Dallas, Texas, USA) was used for drug selection. Proper transgene expression was confirmed by RT-PCR, using primer pairs against human TLR2 (5'-CAGTGGCCAGAAAAGATGAAATA-3'; 5' - GTGGCACAGGACCCCCG - $3^{\prime}$ ) and TLR4 (5'-TGCGGGTTCTACATCAAA- $3^{\prime} ; 5^{\prime}$-CCATCCGAAATTATAAGAAAAGTC- $3^{\prime}$ ) as described (24). All of the TLR transfected cell lines had similar expression of the FLAG epitope (29). Clonal cell lines were also analyzed by $\mathrm{FACS}^{\circledR}$ analysis using specific mAbs against TLR2 and TLR4 (Figure 1). All $\mathrm{CHO}$ cell lines were grown in Ham's F12 medium containing $10 \% \mathrm{FBS}$ and $10 \mu \mathrm{g} / \mathrm{mL}$ of ciprofloxacin, in a humidified, $5 \% \mathrm{CO}_{2}$ environment at $37^{\circ} \mathrm{C}$. Medium was supplemented with $400 \mathrm{U} / \mathrm{mL}$ of hygromycin B and $0.5 \mathrm{mg} / \mathrm{mL}$ of G418 (CHO/CD $14 / \mathrm{moTLR} 2$ and $\mathrm{CHO} / \mathrm{CD} 14 / \mathrm{huTLR} 2)$ or $50 \mu \mathrm{g} / \mathrm{mL}$ of puromycin (CHO/CD14/huTLR4). All of the experiments with $\mathrm{CHO}$ cell lines were performed at least twice, and were confirmed using 2 or more unique clonal cell lines with the same transgenes. CHO/CD14/huTLR4 cell lines exhibited slightly higher constitutive expression of surface CD25 (this appeared to be an effect of the FLAG epitope in minimally activating TLR4-induced

\section{Figure 2}

RSLA blocked LPS-mediated activation in CHO/CD14 reporter cells expressing human TLR4. CHO/CD14 (a and b) and CHO/CD14/huTLR4 (c and d) reporter cells were plated in 24-well dishes. The next day, the cells were exposed to various treatments. (a and c) Cells treated with medium only (stippled lines), RSLA ( $5 \mu \mathrm{g} / \mathrm{mL}$; thick lines), or synthetic lipid IVa $(5 \mu \mathrm{g} / \mathrm{mL})$. (b and d) Cells treated with medium only (stippled lines), LPS $(0.5 \mu \mathrm{g} / \mathrm{mL}$; thin lines) or a combination of LPS and RSLA ( 0.5 and $5 \mu \mathrm{g} / \mathrm{mL}$, respectively) for 20 hours. After harvesting, the cells were stained for surface CD25 and subjected to flow cytometry analysis. Untreated cells (stippled lines) are indicated by " 0 ". The $x$-axis represents relative fluorescence and the $y$-axis represents relative cell number. One representative experiment out of 5 is shown. Note that although basal immunofluorescence in unstimulated $\mathrm{CHO} / \mathrm{CD} 14 / \mathrm{TLR} 4$ cells is slightly higher than in $\mathrm{CHO} / \mathrm{CD} 14$ cells, the $\mathrm{ED}_{50}$ of $\mathrm{CHO} / \mathrm{CD} 14 / \mathrm{TLR} 4$ to LPS-induced reporter activity has been found to be identical (data not shown).
NF- $\mathrm{KB}$ ), but these lines maintained their ability to respond to LPS. Human monocytic THP-1 cells were maintained in RPMI-1640 medium containing 10\% FBS, as described previously (10).

Flow cytometry analysis of NF- $\kappa B$ activity. Cells were plated at a density of $7.5 \times 10^{4}$ cells per well in 24-well dishes. The next day, the cells were stimulated as indicated in Ham's F12 medium containing 10\% FBS (total volume of $0.25 \mathrm{~mL} /$ well). Subsequently, the cells were harvested with trypsin-EDTA and labeled with FITCCD25 mAb. Analysis by flow cytometry was performed as described previously (26).

IL-6 assay. CHO cells were plated at a density of $2 \times 10^{4}$ cells per well in 24-well dishes. The next day, the cells were washed twice with PBS and stimulated with LPS, alone or in combination with RSLA or lipid IVa for 10 hours in RPMI-1640 and 2\% FCS. Cell-free supernatants were harvested, and IL- 6 was measured by the B13.29 cell proliferation bioassay as described elsewhere $(30,31)$.

Cloning of hamster TLR4. PCR primers (5'-TGCTGCCAACATCATCCA and $5^{\prime}$ - ${ }^{\circ}$ TTTTCCATCCAACAGGGCTTTT) were designed based on the sequences of rat and human TLR4 to generate a hamster-specific TLR4 PCR fragment of $304 \mathrm{bp}$ using $\mathrm{CHO} / \mathrm{CD} 14$ cDNA as a template. The PCR fragment was labeled with $\left.{ }^{32} \mathrm{P}\right] \mathrm{CTP}$ and then used to screen a $\mathrm{CHO} / \mathrm{CD} 14$ cDNA library (31). Positive clones were converted into phagemids by single clone excision, and were sequenced using an $\mathrm{ABI} 373 \mathrm{~A}$ automated sequencer (PE Applied Biosystems, Foster City, California, USA). Four splice variants of CHO-TLR4 were identified, 1 of which encoded a full-length, functional pro-

a

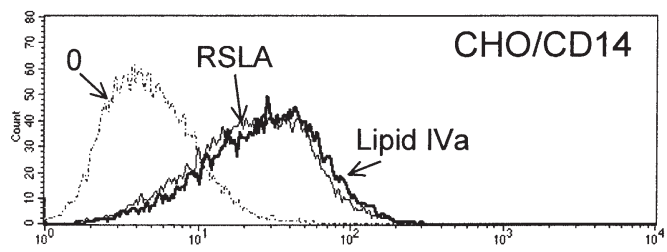

b

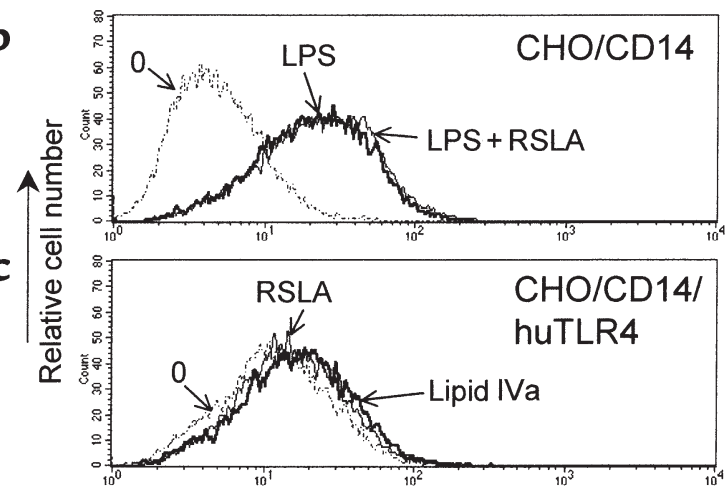

d

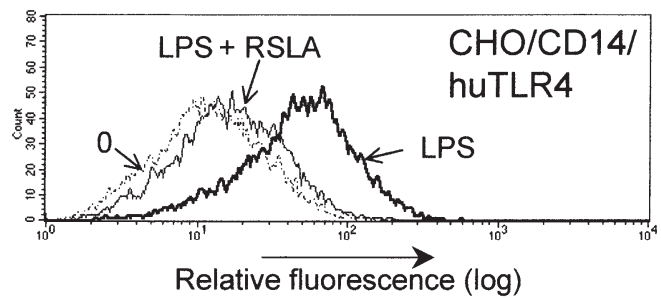


Figure 3

Lipid IVa and RSLA stimulate the release of the cytokine IL- 6 from CD14-expressing hamster fibroblasts but become LPS antagonists when these cells overexpress human TLR4. CHO/CD14 cells and $\mathrm{CHO} / \mathrm{CD} 14 /$ huTLR4 cells were allowed to adhere overnight in 24well dishes. The next day, the cells were exposed to increasing amounts of S. minnesota Re595 LPS in the presence of medium only (diamonds), $0.5 \mu \mathrm{g} / \mathrm{mL}$ lipid IVa or RSLA (squares), or $5 \mu \mathrm{g} / \mathrm{mL}$ of the same compounds (circles) for 10 hours. Supernatants were assayed for IL- 6 by bioassay. Data represents mean values of 3 seperate fractions \pm SD shown is 1 experiment out of 2 performed. tein. Based on the sequence of CHO-TLR4, primers (5'CTCACCCTTAGCCCAGAACATTTT and 5'-TGGGGCTTAGCTCTTTTCCTTCAG) were designed to clone the fulllength TLR4 using the TOPO TA Cloning kit (Invitrogen Corp.), and $P f u$ polymerase from native hamster macrophage cDNA, prepared from mRNA as described previously (24). Hamster TLR4 was subcloned into the $5^{\prime} \mathrm{NotI} / 3^{\prime}$ SalI site of pFLAG-CMV-1 and sequenced.

Transient transfection and NF- $\kappa B$ assay. THP- 1 cells were plated at a density of $2 \times 10^{6}$ cells per well in 6 -well dishes. Cells were transiently cotransfected with $0.5 \mu \mathrm{g}$ of the luciferase reporter plasmid pELAM.luc plus $0.5 \mu \mathrm{g}$ of TLR4 (hToll), pFLAG-hamTLR4, or pcDNA3.1 using Effectene transfection reagent (QIAGEN Inc., Valencia, California, USA). The next day, the cells were stimulated with LPS, lipid IVa, or RSLA for 5 hours. The response to stimulation was measured by assessing luciferase activity in $50 \mu \mathrm{g}$ of total cellular lysate, as described (24).

\section{Results}

TLR4 is expressed in CHO cells and hamster macrophages. Both TLR2 and TLR4 have been implicated in LPS signaling. The identification of Lps as Tlr4 (17-19), and the finding that TLR2-deficient macrophages responded to LPS (24) suggested to us that TLR4 was the principal LPS signal transducer in mammalian cells. We confirmed that CHO-K1 fibroblasts and hamster macrophages expressed full-length TLR4 by cloning and sequencing the cDNA from both cell types. Hamster TLR4 is a type I transmembrane protein with a predicted transmembrane region between amino acids 630 and 650 . The hamster TLR4 amino acid sequence is $79 \%$ and $70 \%$ identical to mouse TLR4 and human TLR4, respectively; the cytoplasmic portions are $94 \%$ and $90 \%$ identical. The sequence of hamster TLR4 reported in this paper is deposited in the GenBank database (accession number AF153676).
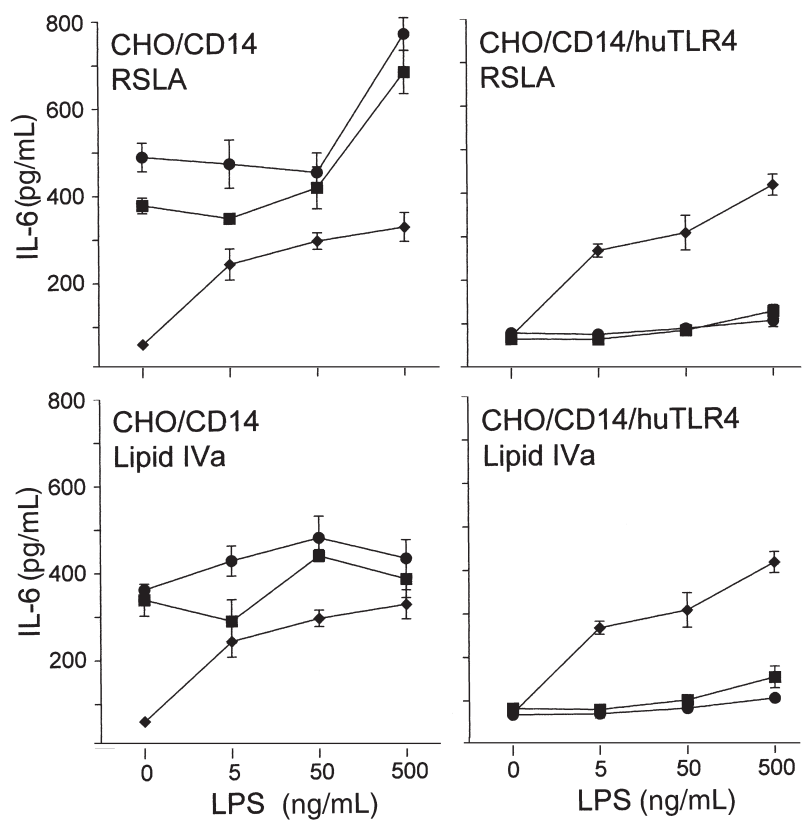

RSLA and lipid IVa, both LPS mimetics in hamster cells, antagonize LPS when tested in CD14-expressing CHO fibroblasts that overexpress human TLR4. We tested CD14-positive CHO fibroblast reporter cell lines that were stably cotransfected with human TLR4 (shown in Figure 1) to determine if overexpression of the human receptor would change the pharmacological phenotype of the cells with respect to their responses to RSLA and lipid IVa.

Mock-transfected CHO cells do not respond to the presence of LPS, but after CD14 transfection, $\mathrm{CHO} / \mathrm{CD} 14$ cells are capable of responding to low concentrations of LPS, RSLA, and lipid IVa (6). For example, LPS, RSLA, and lipid IVa all activated the NF- $\mathrm{kB}$-dependent reporter gene in the singly transfected CHO/CD14 cell line (Figure 2, a and b). No evidence of inhibition or synergy was observed when the cells were coincubated with LPS and RSLA together. In contrast to the $\mathrm{CHO} / \mathrm{CD} 14$ reporter cell line, $\mathrm{CHO} / \mathrm{CD} 14 /$ huTLR4 cells had virtually no response to lipid IVa or RSLA (Figure 2c). LPS retained full stimulatory activity with $\mathrm{CHO} / \mathrm{CD} 14 /$ huTLR 4 cells, whereas incubation of CHO/CD14/huTLR4 with LPS and RSLA together failed to result in cellular activation, consistent with receptor-mediated antagonism of LPS by RSLA (Figure 2d). Dose-dependent inhibition of LPS activation of the reporter gene was also observed in the $\mathrm{CHO} / \mathrm{CD} 14 /$ huTLR4 cell line using lipid IVa (data not shown). As a control, CHO/CD14 cells and $\mathrm{CHO} / \mathrm{CD} 14 /$ huTLR4 cells were exposed to human IL-1 $\beta(5 \mathrm{ng} / \mathrm{mL})$; the 2 cell lines responded nearly identically (9.3- and 9.4-fold enhancement of CD25 expression, respectively; data not shown).

The LPS-inhibiting activities of lipid IVa and RSLA in $\mathrm{CHO} / \mathrm{CD} 14 /$ huTLR4 cells were confirmed by assessing LPS-exposed cells for nuclear translocation of NF-KB by electrophoretic mobility shift assay. 
a

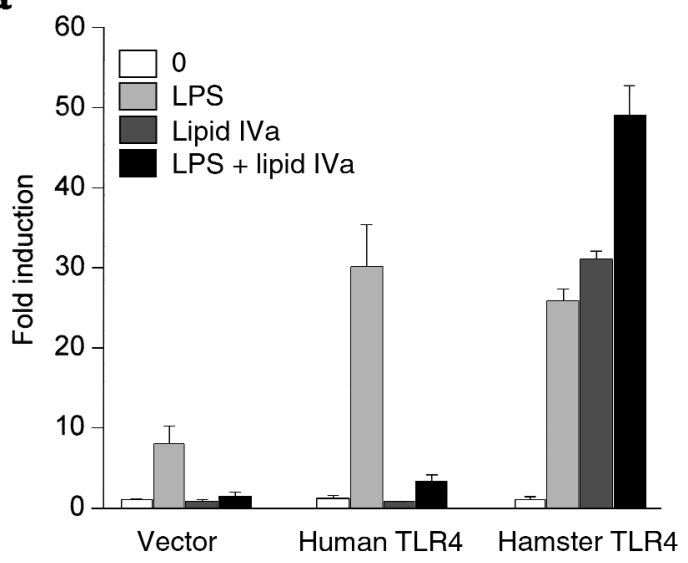

b

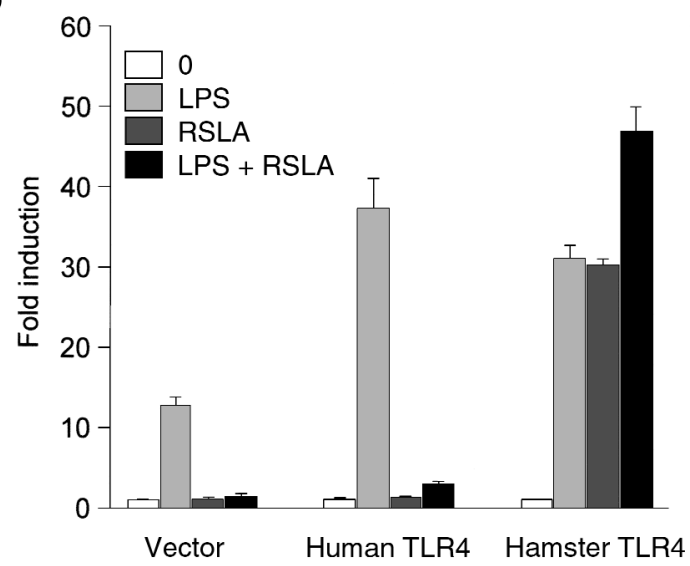

\section{Figure 4}

Reversal of the human phenotype by hamster TLR4 expression: lipid IVa and RSLA activate THP-1 monocytes that overexpress hamster TLR4. THP-1 cells were plated at a density of $2 \times 10^{6}$ cells per well in 6 -well dishes, and then transiently transfected with the reporter plasmid pELAM.luc plus either pcDNA3.1 (vector), hToll (human TLR4), or pFLAG-hamTLR4 (hamster TLR4). The next day, the cells were incubated with medium alone $(0)$, LPS $(10 \mathrm{ng} / \mathrm{mL})$, lipid IVa $(1 \mu \mathrm{g} / \mathrm{mL})$, or a combination of LPS and lipid IVa $(10$ $\mathrm{ng} / \mathrm{mL}$ plus $1 \mu \mathrm{g} / \mathrm{mL}$, respectively) (a), or in a separate experiment with medium alone (0), LPS (10 $\mathrm{ng} / \mathrm{mL}), \mathrm{RSLA}(1 \mu \mathrm{g} / \mathrm{mL}), \mathrm{or}$ a combination of LPS and RSLA ( $10 \mathrm{ng} / \mathrm{mL}$ and $1 \mu \mathrm{g} / \mathrm{mL}$, respectively) (b) for 5 hours. Luciferase activity was measured as described in Methods and plotted as the fold induction of activity compared with vector-transfected, unstimulated controls. The values shown are mean \pm SD of triplicate transfections in 1 representative experiment out of 3 . Similar results were observed when cells were stimulated with $100 \mathrm{ng} / \mathrm{mL}$ LPS and a 10-fold excess of inhibitor (data not shown).

Again, all of the cell lines responded nearly identically to IL-1 $\beta$ (data not shown). Hence, with heterologous overexpression of human TLR4 in a hamster cell, the LPS-specific pharmacology of the cells appeared to be altered from a hamster to a human phenotype.

We next tested lipid IVa and RSLA for their ability to antagonize LPS-induced release of the inflammatory cytokine IL-6, as another indicator of Toll receptor function. As expected, LPS, lipid IVa, and RSLA all strongly stimulated $\mathrm{CHO} / \mathrm{CD} 14$ cells to produce IL6 (Figure 3). In contrast, CHO/CD14/huTLR4 cells exhibited a human phenotype: both lipid IVa and RSLA were potent inhibitors of LPS-induced IL-6 release in the cells expressing the human transgene (Figure 3). Thus, the cells expressing human TLR4 acquired the ability to recognize lipid IVa and RSLA

\section{Figure 5}

Expression of human TLR2 in CHO/CD14 cells does not change the responses to lipid IVa and synthetic lipid A. Untransfected CHO/CD14 reporter cells (a) or $\mathrm{CHO} / \mathrm{CD} 14$ reporter cells stably transfected with human TLR2 (29) (b) were stimulated with synthetic lipid A (0.5 $\mu \mathrm{g} / \mathrm{mL})$ as a positive control, or with lipid IVa $(0.5 \mu \mathrm{g} / \mathrm{mL})$ as indicated. After 20 hours, the cells were harvested and stained for surface CD25 and then subjected to flow cytometry analysis. Untreated cells (stippled lines) are indicated by " 0 ". The $x$-axis represents relative fluorescence, and the $y$-axis represents relative cell number. Results shown are from 1 of 2 experiments performed. Similar results were observed in RSLA-exposed cells and with $\mathrm{CHO} / \mathrm{CD} 14 /$ moTLR2 cells treated with either lipid IVa or RSLA. as LPS antagonists, with respect to LPS-induced release of IL-6.

The LPS inhibitors lipid IVa and RSLA become LPS mimetics when tested in buman monocytes that express hamster TLR4. Based on the above results, we predicted that if hamster TLR4 were overexpressed in human cells, the hamster-defined pharmacology would predominate in cells exposed to lipid IVa or RSLA. We chose the human cell line THP-1 to test because the ability of lipid IVa and RSLA to inhibit LPS in these cells has already been established (10).

THP-1 cells were cotransfected with the structural gene for human TLR4, hamster TLR4, or a control plasmid, plus an NF- $\kappa B-d e p e n d e n t ~ l u c i f e r a s e$ reporter construct (pELAM.luc; ref. 20). After allowing 24 hours for transgene expression to occur, the

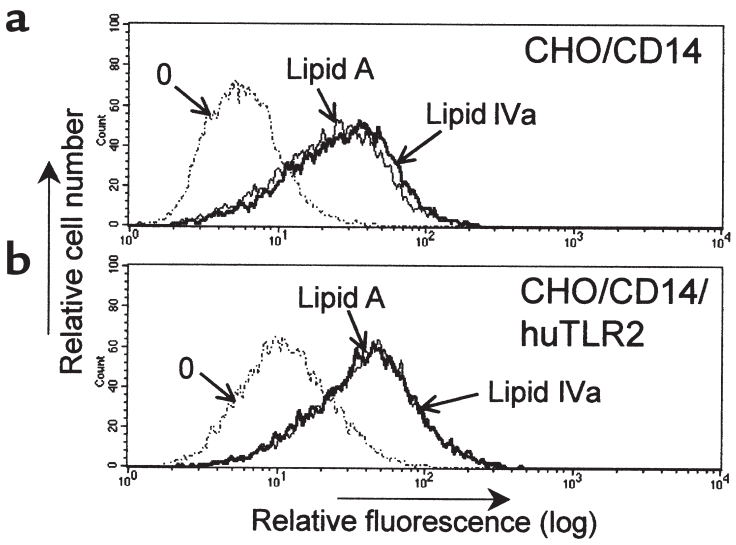


cells were tested for their responses to LPS, lipid IVa (Figure 4a), and RSLA (Figure 4b). Similar to our previous experiments with THP-1 monocytes, cells transfected with vector alone had no response to lipid IVa or RSLA, but responded strongly to LPS $(\sim 8$-fold enhancement of luciferase activity). This response to LPS was inhibited by coincubation with lipid IVa and LPS together. When cells were transfected with human TLR4, the response to LPS was augmented to nearly 30 -fold above background, a response that was inhibited almost completely by lipid IVa or RSLA. In complete contrast to huTLR4-expressing cells, hamster TLR4-transfected THP-1 monocytes experienced similar degrees of stimulatory activity in response to LPS, lipid IVa, and RSLA. When cells were exposed to LPS plus lipid IVa or LPS plus RSLA, stimulated activity was slightly additive. Thus, expression of hamster TLR4 in human cells imparts the characteristic hamster LPS pharmacology.

TLR2 does not mediate species-specific responses to lipid IVa or RSLA. TLR2 has been identified as an LPS signal transducer in transfected cell lines $(15,16)$. We previously reported that $\mathrm{CHO} / \mathrm{CD} 14$ cells do not express TLR2 (24). Thus, unlike with TLR4, we had the ability to test human TLR2 function independently of the activity of endogenous hamster TLR2. We hypothesized that if TLR 2 could account for species-specific recognition of lipid A-like molecules, then overexpression of TLR2 in CHO/CD14 cells should also result in an alteration of the phenotype with respect to LPS antagonists.

As expected, LPS, synthetic lipid A, and lipid IVa functioned as LPS agonists in CHO/CD14 cells (Figure 5a). However, unlike overexpression of human TLR4, overexpression of human TLR2 in $\mathrm{CHO} / \mathrm{CD} 14$ cells (Figure 1) did not noticeably alter the LPS-agonist response to lipid IVa (Figure 5b). Similar observations were made with RSLA (not shown). Although RSLA inhibits LPS in mouse macrophages (10), it also failed to inhibit LPS responses in $\mathrm{CHO} / \mathrm{CD} 14 / \mathrm{moTLR} 2$ cells (data not shown). Thus, expression of TLR2 from 2 different mammalian species did not change the responses of hamster cells to lipid IVa or RSLA. To support our findings, expression of mouse TLR 2 in human cells did not change the ability of RSLA to function as an LPS antagonist (not shown). We conclude that TLR4, and not TLR2, is the major recognition molecule mediating the responses to both LPS and these lipid A-like structures.

\section{Discussion}

Reports that both TLR2 and TLR4 are capable of functioning as LPS signal transducers have significantly enhanced our understanding of how LPS engagement of $\mathrm{CD} 14$ results in productive signal transduction. At the same time, however, these reports are confusing. Are both TLRs LPS signal transducers? Is TLR2 a signal transducer in humans (as evidenced by work with human TLR2 in human HEK293 cells), whereas TLR4 is the signal transducer in mice, accounting for the LPS hyporesponder phenotype of $\mathrm{C} 3 \mathrm{H} / \mathrm{HeJ}-$, C57BL10/ScCr-, and TLR4knockout mice? Does TLR2 have another function aside from its role as an LPS signal transducer that better defines its importance?

Although this study does not entirely answer these questions, it clearly supports the notion that the principal LPS signal transducer is TLR4 in all mammalian species. There is little reason to suppose that the LPS signal transducer in man is fundamentally different from that in mice or hamsters. Rather, TLR4 appears to be the dominant LPS receptor in all species, and its expression dictates the heretofore puzzling species-specific pharmacology of the LPS antagonists, including lipid IVa and RSLA.

Although the failure to observe TLR2-mediated, species-specific pharmacology does not rule out the importance of this receptor in LPS signal transduction, other recent observations suggest that TLR2 is of limited importance for the response of quiescent native cells to LPS. First, TLR4 mutant mice are extremely hyporesponsive to LPS (17-19). There is no reason to believe that these mice harbor a mutation in TLR2 (this is especially true for the TLR4-knockout mouse, whose genetic lesion has been entirely defined). Conversely, peritoneal macrophages from Chinese hamsters do not express a functional full-length mRNA for TLR2, yet are sensitive to low concentrations of LPS $(6,24)$. As reported here, these macrophages express a full-length, functional transcript for TLR4. Thus, in contrast to TLR4, TLR2 expression is not required for sensitive responses to LPS.

We and others have observed that TLR2-transfected cells, but not TLR4 transfectants, respond to a diverse number of bacteria and bacterial products, including Gram-positive organisms $(22,29,32)$, spirochetes $(22$, $33,34)$, mycoplasma (22), and mycobacteria (22, 34-36). LPS may have structural features in common with components of these bacteria that enable it to activate TLR2. The reason that TLR2 is unable to compensate for the TLR4 defect in C3H/HeJ-, C57BL10/ScCr-, and TLR4knockout mice is unclear, but this probably reflects inadequate receptor density or a reduced activation state in quiescent macrophages. Alternatively, TLR2 may be a low-affinity LPS receptor in vivo. Although LPS responses involving TLR2 may be limited to transfected cell lines, we note that chronically infected $\mathrm{C} 3 \mathrm{H} / \mathrm{HeJ}$ mice have been reported to die from injections of purified LPS (37), an effect that may be due to altered activity of a Toll receptor other than TLR4.

Some investigators believe that the mechanism of activation of TLR 4 is probably similar to the mechanism by which the Toll receptor is activated in Drosophila (12, 38, 39). In this fly, the spätzle gene encodes a secreted protein that requires proteolytic activation in order to generate a peptide ligand for Toll. The analogous theory for TLR4, elucidated in a recent review (40), proposes that a comparable, evolutionarily conserved proteolytic processing reaction generates a peptide ligand for TLR4 as 
a result of the direct interaction of endotoxin with an LPS-sensitive protease. Despite the attractiveness of this theory, it is difficult to conceive how the differential activation or inhibition of a protease by lipid IVa or RSLA could account for the species-specific pharmacological observations presented in this report. Although a peptide ligand may exist for TLR4, we conclude that the Toll/spätzle paradigm is not likely to hold for LPS signal transduction in mammals.

The most likely explanation of the mechanism of LPS inhibition by lipid IVa and RSLA is that these antagonists compete with the lipid A portion of LPS for a common binding site on TLR4. It is notable that direct ligand binding studies that demonstrate the validity of this statement have not been reported. However, such binding studies may require a considerable amount of effort to complete. LPS is a notoriously difficult ligand with which to work, because it is difficult to radiolabel to achieve high specific activity and does not form monomers in aqueous suspension because of its amphipathicity. Furthermore, the binding of LPS to TLR4 may occur in the transmembrane or cytoplasmic portion of the molecule. Most LPS-responsive cells coexpress CD14, so the differences in the way LPS binds to TLR4 and to CD14 may be difficult to demonstrate. Thus, the demonstration of LPS binding to TLR4 may present difficult technical problems. It is interesting that despite extensive genetic data demonstrating the interactions of Drosopbila Toll with its ligand, a report of Toll/spätzle binding has not yet been published. As a result of the absence of such binding studies, alternative explanations of TLR4 function have been proposed. Wright has recently suggested that direct binding of LPS to TLR4 may not occur. He proposes that instead, TLR4 may function as a membrane "sensor," responding to alterations in the colligative properties of mammalian membranes resulting from CD14-mediated LPS internalization (41). Our experiments are not entirely in conflict with this point of view, although only the establishment of a ligand-binding assay can lend credence to or disprove this novel hypothesis.

We believe that a molecular genetic approach to the identification of the LPS-binding domain of TLR4 may prove helpful. Human, mouse, and hamster cells each have a unique profile of pharmacological responses to lipid IVa and RSLA, suggesting that a region of nonidentity in TLR4 accounts for the species-specific responses to these compounds. By combining the tools of molecular genetics with LPS pharmacology, a critical region of TLR4 can be identified for further studies, such as the crystallization and resolution of the 3dimensional structure of the putative LPS-binding domain in the presence of LPS or its inhibitors.

The mortality due to Gram-negative sepsis remains a serious problem in the world today, and the challenges ahead are intimidating. Several carefully designed pharmaceutical approaches to modifying the clinical outcome of sepsis have failed. As with any illness, a true understanding of the pathophysiology of disease is a critical step in designing effective remedies. The identification of TLR4 as a target for LPS-mediated diseases should lead to renewed optimism that effective therapies against this heretofore enigmatic disorder ultimately will be achieved.

\section{Acknowledgments}

This work was supported by The Research Council of Norway and the Norwegian Cancer Society (E. Lien), the Medical Research Service of the Department of Veterans Affairs (N. Qureshi), and by National Institutes of Health Grants GM-54060, AI-38505, AI-01476 (A. Yoshimura, H. Heine, R.R. Ingalls, and D.T. Golenbock), HL-55681 (M.J. Fenton), GM-50870 (N. Qureshi), and AI-31628-08 (R.W. Finberg).

1. Sands, K.E., et al. 1997. Epidemiology of sepsis syndrome in 8 academic medical centers. Academic Medical Center Consortium Sepsis Project Working Group. JAMA. 278:234-240.

2. Raetz, C.R., et al. 1991. Gram-negative endotoxin: an extraordinary lipid with profound effects on eukaryotic signal transduction. FASEB $J$. 5:2652-2660.

3. Fenton, M.J., and Golenbock, D.T. 1998. LPS-binding proteins and receptors. J. Leukoc. Biol. 64:25-32.

4. Kitchens, R.L., and Munford, R.S. 1995. Enzymatically deacylated lipopolysaccharide (LPS) can antagonize LPS at multiple sites in the LPS recognition pathway. J. Biol. Chem. 270:9904-9910.

5. Kitchens, R.L., Ulevitch, R.J., and Munford, R.S. 1992. Lipopolysaccharide (LPS) partial structures inhibit responses to LPS in a human macrophage cell line without inhibiting LPS uptake by a CD14-mediated pathway. J. Exp. Med. 176:485-494.

6. Delude, R., et al. 1995. CD14 enhances cellular responses to endotoxin without imparting ligand-specific recognition. Proc. Natl. Acad. Sci. USA. 92:9288-9292.

7. Ingalls, R.R., Monks, B.G., and Golenbock, D.T. 1999. Membrane expression of soluble endotoxin-binding proteins permits lipopolysaccharide signaling in Chinese hamster ovary fibroblasts independently of CD14. J. Biol. Chem. 274:13993-13998.

8. Ulevitch, R.J., and Tobias, P.S. 1994. Recognition of endotoxin by cells leading to transmembrane signaling. Curr. Opin. Immunol. 6:125-130.

9. Lynn, W.A., and Golenbock, D.T. 1992. Lipopolysaccharide antagonists. Immunol. Today. 13:271-276.

10. Golenbock, D.T., Hampton, R.Y., Qureshi, N., Takayama, K., and Raetz, C.R.H. 1991. Lipid A-like molecules that antagonize the effects of endotoxins on human monocytes. J. Biol. Chem. 266:19490-19498.

11. Belvin, M.P., and Anderson, K.V. 1996. A conserved signaling pathway: the Drosophila Toll-dorsal pathway. Annu. Rev. Cell Dev. Biol. 12:393-416.

12. Lemaitre, B., Nicolas, E., Michaut, L., Reichhart, J.M., and Hoffmann, J.A. 1996. The dorsoventral regulatory gene cassette spatzle/Toll/cactus controls the potent antifungal response in Drosophila adults. Cell. 86:973-983.

13. Williams, M.J., Rodriguez, A., Kimbrell, D.A., and Eldon, E.D. 1997. The 18 -wheeler mutation reveals complex antibacterial gene regulation in Drosophila host defense. EMBO J. 16:6120-6130.

14. Rock, F.L., Hardiman, G., Timans, J.C., Kastelein, R.A., and Bazan, J.F. 1998. A family of human receptors structurally related to Drosophila Toll. Proc. Natl. Acad. Sci. USA. 95:588-593.

15. Yang, R.B., et al. 1998. Toll-like receptor-2 mediates lipopolysaccharideinduced cellular signalling. Nature. 395:284-288.

16. Kirschning, C.J., Wesche, H., Ayres, T.M., and Rothe, M. 1998. Human Toll-like receptor 2 confers responsiveness to bacterial lipopolysaccharide. J. Exp. Med. 188:2091-2097.

17. Poltorak, A., et al. 1998. Defective LPS signaling in $\mathrm{C} 3 \mathrm{H} / \mathrm{HeJ}$ and C57BL/10ScCr mice: mutations in Tlr4 gene. Science. 282:2085-2088.

18. Hoshino, K., et al. 1999. Toll-like receptor 4 (TLR4)-deficient mice are hyporesponsive to lipopolysaccharide: evidence for TLR4 as the Lps gene product. J. Immunol. 162:3749-3752.

19. Qureshi, S.T., et al. 1999. Endotoxin-tolerant mice have mutations in Toll-like receptor 4 (Tlr4). J. Exp. Med. 189:615-625.

20. Chow, J.C., Young, D.W., Golenbock, D.T., Christ, W.J., and Gusovsky, F. 1999. Toll-like receptor-4 mediates lipopolysaccharide-induced signal transduction. J. Biol. Chem. 274:10689-10692.

21. Liu, W.-C., Oikawa, M., Fukase, K., Suda, Y., and Kusumoto, S. 1999. A divergent synthesis of lipid A and its chemically stable unnatural analogues. Bull. Chem. Soc. Jpn. 72:1377-1385.

22. Lien, E., et al. 1999. Toll-like receptor 2 functions as a pattern recogni- 
tion receptor for diverse bacterial cell wall products. J. Biol. Chem. 274:33419-33425.

23. Shimazu, R. et al. 1999. MD-2, a molecule that confers lipopolysaccharide responsiveness on Toll-like receptor 4. J. Exp. Med. 189:1777-1782.

24. Heine, H., et al. 1999. Cutting edge: cells that carry a null allele for Tolllike receptor 2 are capable of responding to endotoxin. J. Immunol. 162:6971-6975.

25. Medzhitov, R., Preston-Hurlburt, P., and Janeway, C.A., Jr. 1997. A human homologue of the Drosophila Toll protein signals activation of adaptive immunity. Nature. 388:394-397.

26. Delude, R.L., Yoshimura, A., Ingalls, R.R., and Golenbock, D.T. 1998 Construction of a lipopolysaccharide reporter cell line and its use in identifying mutants defective in endotoxin, but not TNF-alpha, signal transduction. J. Immunol. 161:3001-3009.

27. Schindler, U., and Baichwal, V.R. 1994. Three NF-kappa B binding sites in the human E-selectin gene required for maximal tumor necrosis factor alpha-induced expression. Mol. Cell. Biol. 14:5820-5831.

28. Golenbock, D., Liu, Y., Millham, F., Freeman, M., and Zoeller, R. 1993 Surface expression of human CD14 in Chinese hamster ovary fibroblasts imparts macrophage-like responsiveness to bacterial endotoxin. J. Biol. Chem. 268:22055-22059.

29. Yoshimura, A., et al. 1999. Cutting edge: recognition of gram-positive bacterial cell wall components by the innate immune system occurs via Toll-like receptor 2. J. Immunol. 163:1-5.

30. Aarden, L.A., De Groot, E.R., Schaap, O.L., and Lansdorp, P.M. 1987. Production of hybridoma growth factor by human monocytes. Eur. J. Immunol. 17:1411-1416.

31. Heine, H., Delude, R.L., Monks, B.G., Espevik, T., and Golenbock, D.T 1999. Bacterial lipopolysaccharide induces expression of the stress response genes hop and H411. J. Biol. Chem. 274:21049-21055.
32. Schwandner, R., Dziarski, R., Wesche, H., Rothe, M., and Kirschning, C.J 1999. Peptidoglycan- and lipoteichoic acid-induced cell activation is mediated by Toll-like receptor 2. J. Biol. Chem. 274:17406-17409.

33. Hirschfeld, M., et al. 1999. Cutting edge: inflammatory signaling by Borrelia burgdorferi lipoproteins is mediated by Toll-like receptor 2. J. Immunol. 163:2382-2386.

34. Brightbill, H.D., et al. 1999. Host defense mechanisms triggered by microbial lipoproteins through Toll-like receptors. Science. 285:732-736.

35. Means, T.K., et al. 1999. The CD14 ligands lipoarabinomannan and lipopolysaccharide differ in their requirement for Toll-like receptors. $J$. Immunol. 163:6748-6755.

36. Means, T.K., et al. 1999. Human Toll-like receptors rediate cellular activation by M. tuberculosis. J. Immunol. 163:3920-3927.

37. Vogel, S.N., Moore, R.N., Sipe, J.D., and Rosenstreich, D.L. 1980. BCGinduced enhancement of endotoxin sensitivity in $\mathrm{C} 3 \mathrm{H} / \mathrm{HeJ}$ mice. I. In vivo studies. J. Immunol. 124:2004-2009.

38. DeLotto, Y., and DeLotto, R. 1998. Proteolytic processing of the Drosophila Spatzle protein by easter generates a dimeric NGF-like molecule with ventralising activity. Mech. Dev. 72:141-148.

39. Morisato, D., and Anderson, K.V. 1994. The spatzle gene encodes a component of the extracellular signaling pathway establishing the dorsalventral pattern of the Drosophila embryo. Cell. 76:677-688.

40. Medzhitov, R., and Janeway, C.A., Jr. 1998. Self-defense: the fruit fly style. Proc. Natl. Acad. Sci. USA. 95:429-430.

41. Wright, S.D. 1999. Toll, a new piece in the puzzle of innate immunity. J. Exp. Med. 189:605-609.

42. Lynn, W.A., Raetz, C.R., Qureshi, N., and Golenbock, D.T. 1991 Lipopolysaccharide-induced stimulation of CD11b/CD18 expression on neutrophils. Evidence of specific receptor-based response and inhibition by lipid A-based antagonists. J. Immunol. 147:3072-3079. 